\title{
MedienPädagogik
}

Zeitschrift für Theorie und Praxis der Medienbildung

\section{Konzeption und Durchführung der Evaluation einer virtuellen Lernumgebung:}

\author{
Das Projekt Methodenlehre-Baukasten
}

Rolf Schulmeister, Burkhard Vollmers, Robert Gücker und Klaus Nuyken

\section{Einführung}

Die Universität Hamburg implementiert zusammen mit anderen Partneruniversitäten ein E-Learning-Programm, das in der Methodenlehre und Statistikausbildung eingesetzt werden soll. Dieses Programm, der Methodenlehre-Baukasten (MLBK), verfolgt ein didaktisches Konzept, das sich an der kognitivkonstruktivistischen Lerntheorie von Jean Piaget orientiert und sich an Jerome Bruners Konzept des entdeckenden Lernens anlehnt. Dieses didaktische Modell ist für die Zielgruppe des MLBK, Studierende der Sozialwissenschaften in den Anfangssemestern mit Ängsten und Desinteresse gegenüber Statistik (Statistik-Angst, s.u.), unserer Annahme nach besonders gut geeignet.

Eingesetzt wird der MLBK an den vier beteiligten Universitäten Hamburg, Bremen, Rostock und Greifswald in den grundständigen Studiengängen Psychologie, Erziehungswissenschaften und Soziologie. Im Laufe der Evaluation gilt es zunächst, aus der Stichprobe aller teilnehmenden Studierenden diejenigen zu identifizieren, die unsere Zielgruppe ausmachen. Das geschieht mit Hilfe eines Fragebogens, der die Statistik-Angst misst. Als weiteres Merkmal zur Bestimmung der Zielgruppe erfolgt eine Gruppierung der untersuchten Personen vorab gemäß ihrer kognitiven Lernstile. Die Evaluation des MLBK untersucht systematisch, welche Lernchancen die Zielgruppe durch das Lernsystem erhält. Deshalb wird der Mehrwert des MLBK nicht global und kontextfrei ermittelt, sondern in Relation zu den Bedürfnissen der angestrebten Zielgruppen.

Insgesamt wird im Evaluationsdesign die Interaktion folgender vier Konstrukte und die Differenzierung der Stichprobe in diesen vier Konstrukten untersucht:

- Lernvoraussetzungen

- Usability und Zufriedenheit

- Motivation und Statistik-Angst

- Kognitive Lernstile.

Ermittelt werden Differenzierungen zwischen den Gruppen durch die Variable subjektive Lernzufriedenheit (und Usability) und die Variable objektiver Lern- 
erfolg. Bevor das Evaluationsdesign ausführlich dargestellt wird, steht im Folgenden zunächst die Didaktik im Mittelpunkt. So wird deutlich, was unter Statistik-Angst verstanden wird und welche kognitiven Lernstile der didaktische Ansatz des MLBK besonders anspricht. ${ }^{1}$

\section{Die zielgruppenspezifische Didaktik des Methodenlehre-Baukastens (MLBK)}

Der MLBK richtet sich in erster Linie an Studierende der Geistes- und Sozialwissenschaften in den ersten Semestern. Diese Zielgruppe zeigt erfahrungsgemäß häufig eine starke Abneigung, Statistik als Hilfswissenschaft für ihr Fach zu lernen. Ihre Lernschwierigkeiten im Fach Statistik beruhen größtenteils auf fehlender Motivation, da etlichen Studierenden anfangs der Brückenschlag zwischen Statistik und ihrem Fach nicht gelingt. Diese ungünstige Ausgangslage verstärkt sich durch ungeeignete traditionelle Lehrmethoden. Mit der Vorgabe von mathematischen Formeln und Definitionen durch Statistikexperten in den Rollen der Lehrenden wird oftmals auf einem zu hohen formalabstrakten Denkniveau angesetzt. Die auf unmittelbarer Anschauung beruhenden Kognitionen und Erwartungen der Studienanfänger werden nicht ausreichend berücksichtigt (Schulmeister 1983). Den Lernenden bleibt die Möglichkeit der Eigenstrukturierung der Lerninhalte verwehrt, so dass für sie der Zusammenhang der Statistik mit anderen Inhalten ihres Studienfaches immer weiter aus dem Blick gerät und sich die Abneigung, Statistik zu lernen, weiter erhöht. Verschiedene empirische Untersuchungen haben diesen spezifischen Lernwiderstand belegt. Das Konstrukt „Statistik-Angst“ stammt aus den USA (Dreger/Aiken 1957) und wurde in Deutschland besonders unter Studienanfängern beschrieben und empirisch untersucht, zunächst in der Psychologie (Heemskerk 1975; Schulmeister 1983), später in der Pädagogik (Renkl 1994; Abel 1999).

Das didaktische Konzept des Methodenlehre-Baukastens hat zum Ziel, erfolgreich zum Abbau von negativen Einstellungen gegenüber dem Statistiklernen beizutragen und Verbindungen zum Fachinteresse der Studierenden herzustellen. Der MethodenlehreBaukasten hat deshalb zwei konzeptionelle Leitmotive. Das erste Motiv ist die Förderung der Motivation der Studierenden durch Koppelung der Lektionen und Übungen an fachspezifische empirische Untersuchungen. Vorbild für dieses Konzept sind problemorientierte Multimedia- Programme in der Medizin (Schulmeister 2002a), mit dem Unterschied, dass, entsprechend der Fokussierung auf Forschungsmethoden und Statistik statt auf medizinische Diagnosen an Einzelfällen, bevölkerungsrepräsentative empirische

1 Dieser Aufsatz geht auf einen Vortrag zurück, den Robert Gücker am 27.11.2003 in Magdeburg auf der Herbsttagung der Sektion Medienpädagogik der DGfE gehalten hat. Das Korreferat stammte von Dr. Ulf Ehlers, dessen wertvolle Hinweise, ebenso wie einige Anregungen aus der nachfolgenden Diskussion, in diesen Aufsatz eingeflossen sind. 
Studien das Lernfeld darstellen. In den Erziehungswissenschaften, für deren Module das Interdisziplinäre Zentrum für Hochschuldidaktik in Hamburg (IZHD) verantwortlich zeichnet, ist dies die populäre Shell-Jugendstudie (Jugendwerk der Deutschen Shell 1997). Deren Fragestellungen fließen durchgängig in die Lektionen der deskriptiven und prüfenden Statistik ein. Die anderen Disziplinen übernehmen die Struktur der Lektionen und Übungen von Statistik I und II so, wie sie von den Erziehungswissenschaften erstellt wurden, verwenden aber empirische Studien aus ihrem jeweiligen Gebiet.

Das zweite didaktische Leitmotiv ist das Modell des entdeckenden Lernens als Orientierungspunkt für die Autoren bei der Entwicklung der Lektionen. Dieses Modell geht auf kognitionspsychologische Ideen von Jerome Bruner in den frühen 1960er Jahren zurück (Bruner 1961). Im deutschen Sprachraum wurde es besonders nach der Veröffentlichung des Sammelbandes von Neber (1975) bekannt, der Übersetzungen amerikanischer Aufsätze enthält. Bruner unterstützte die Problemlösefähigkeiten von Schülern, indem er diese selbstständig Lösungen entdecken ließ. Die eigene Entdeckung stellt Bruner zufolge einen Lernprozess dar, in dem die Lernenden ihr Wissen durch eigene Aktivitäten aufbauen. Sie benutzen ihre Vorkenntnisse zu einem Lerngegenstand, um neue Fakten und Zusammenhänge zu suchen und im Hinblick auf eine Lösung zu organisieren.

Dabei gehen die Personen im Idealfall in kleinen kognitiven Schritten vor. Der Lernprozess beginnt bei, aus Sicht der Lernenden, gänzlich neuen Bereichen häufig mit dem Prinzip von Trial and Error. Diese Lernstrategie mündet allmählich in überwiegend Hypothesen überprüfende Verfahren. Der Lernprozess ist insgesamt dadurch gekennzeichnet, dass die Person für sich selbst einen Weg von ihren Vorkenntnissen (naiven Konzepten) zu den möglichen Lösungen (wissenschaftlichen Konzepten) findet (vgl. Abbildung 1). Entdecken bezieht sich somit auf konkrete physische Aktivitäten (wie z.B. Modifizieren von Objekten) und begleitende kognitive Prozesse. Im Ergebnis kommt es zu Umstrukturierungen und Weiterentwicklungen im systematischen Wissen der lernenden Person über den von ihr untersuchten Gegenstandsbereich.

Damit sind die internen Voraussetzungen, die jemand für entdeckendes Lernen benötigt, Umrissen: Der Lernende muss über Vorwissen und Problemlöse-

Kleinste kognitive Schritte:

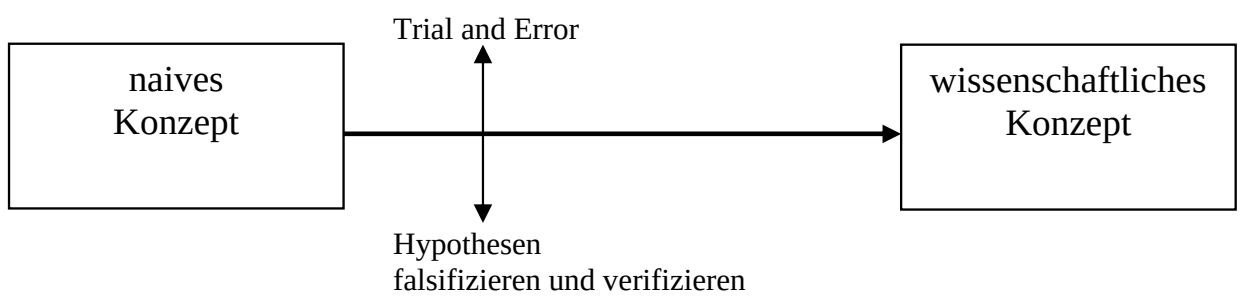

Abb. 1: Schrittweise Konstruktion des wissenschaftlichen Konzepts. 
Fähigkeiten verfügen und imstande sein, selbst organisiert zu lernen. Eine hohe Motivation ist unabdingbare Voraussetzung. Die Motivation wird aber in Rückkoppelung mit dem Programm durch selbst hergestellte, an das individuelle Vorwissen angepasste Lernerfolge auch wieder gefördert (vgl. Heller 1990).

Die Lernziele bauen in den Lektionen aufeinander auf, so dass schrittweises Lernen möglich ist. Erklärungen und Zusammenfassungen, die den Übungen nachfolgen, sorgen dafür, dass die Lernenden die Lernziele auch erreichen können, wenn sie eine Lektion komplett durcharbeiten. Beim MLBK ist kein Mentor oder Teletutor anwesend, um die Lernenden zu unterstützen. Wenn die Lernenden eine Übung durchspielen, so treffen sie häufig auf zu dem jeweiligen Lernziel hinführende Rückmeldungen, die so ähnlich wohl auch ein Mentor im Klassenraum geben würde. Die Übungen mit dem dazugehörigen Feedback zu gestalten, ist für die Autoren keine triviale Aufgabe, müssen doch möglichst viele Alternativen und Lernwege im vorhinein gefunden und durchdacht werden.

Wie die Autoren konkret beim Design von Lektionen und Übungen Vorgehen, um den Nutzern später auch tatsächlich entdeckendes Lernen zu ermöglichen und nicht andere Lernformen zu forcieren, wurde an anderer Stelle ausführlich anhand von Übungsbeispielen beschrieben (vgl. Gücker/Nuyken/Vollmers 2003). Ein expositorisch-definitorischer Darstellungsstil, wie in Statistik-Lehrbüchern üblich, verträgt sich auf keinen Fall mit Bruners Konzept. Ebenso wird vermieden, Übungen als Mittel zum Trainieren, Abfragen und Auswendiglernen einzusetzen. Um konsequent die Lernerperspektive zu berücksichtigen, haben explorative interaktive Übungen im Lernprogramm als Möglichkeiten des selbst gesteuerten Eingriffs des Lerners in das Programm ein deutlich größeres Gewicht als theoretische Erklärungen. Über die Manipulation mit den Lernobjekten soll sich sukzessive das Wissen des Lerners, angepasst an das individuelle Vorwissen, entwickeln. Die Bedeutung der Selbstkonstruktion von Wissen durch aktives Ausprobieren für die kognitive Entwicklung hat insbesondere Jean Piaget in seiner genetischen Epistemologie (Piaget 1970) immer wieder untersucht. Gerade von seinen konstruktivistischen Ideen profitieren die Autoren im MLBK in besonderer Weise. Der MLBK zählt deshalb zu den konstruktivistischen Lernumgebungen (vgl. Moallem 2001 hinsichtlich der Designrichtlinien für konstruktivistische Lernsoftware).

\section{Evaluationsforschung in der Pädagogik, Psychologie und Didaktik}

Die Evaluationsforschung im MLBK steht vor der Herausforderung, die Wirkung und Beurteilung des MLBK auf die weit gefassten Ideen von Bruner und Piaget zu beziehen und gleichzeitig spezifische Wirkungen von Lernobjekten im Sinne klassischer Standards der pädagogischen Evaluationsforschung zu messen.

Gegenstand der empirischen pädagogisch-didaktischen Wirkungsforschung ist die Wirkung spezieller Inhalte, Lernformen, didaktischer Konzepte oder Lehrstile auf Lernerfolg, Zufriedenheit und Motivation derjenigen Personen, 
die als Teilnehmer bzw. Lerner an Bildungs- und Trainingsangeboten partizipieren. An dieses Grundmodell der empirischen Evaluationsforschung ist auch das Evaluationsdesign von Lernsoftware angelehnt. Die methodischen Problemfelder der Evaluation von Präsenzunterricht oder von Beratungs- und Trainingsmaßnahmen in der Pädagogik einerseits sowie der Evaluation von verschiedenen Lernszenarien, virtuellen Lernumgebungen und Programmen in der empirischen Mediendidaktik andererseits sind im Wesentlichen die gleichen, auch wenn sich die didaktischen Rahmenbedingungen, vor allem aus Lernersicht, unterscheiden.

Die Rolle des Lehrers im Präsenzunterricht, der einem didaktischen Konzept und/ oder einem persönlichen Lehrstil folgt, übernimmt beim selbst gesteuerten E-Learning, wenn jemand vor dem Computer Lektionen durcharbeitet, das Computerprogramm. In diesem sind bestimmte didaktische Konzepte vergegenständlicht - in der Art der Präsentation des Stoffs, in der Ansprache des Lerners, in Ausmaß und Form der Interaktivität sowie insgesamt den Möglichkeiten des Eingriffs des Lerners in den Ablauf der Software. Es ist dabei sekundär, ob das jeweilige Lernprogramm mit einem bestimmten, explizit ausformulierten didaktischen Anspruch konzipiert wurde oder nicht. In jedem Programm werden bei genauerer Prüfung unter didaktischen Gesichtspunkten gewisse pädagogische Leitlinien sichtbar.

\subsection{Das Dilemma der Evaluationsforschung: Die Nivellierung von Unterschieden und die unendliche Vielzahl von Lernervariablen}

Die pädagogische Evaluationsforschung ist seit ihrem Beginn in den USA in der ersten Hälfte des letzten Jahrhunderts am Design des psychologischen Experiments mit Versuchs- und Kontrollgruppe orientiert. Als unabhängige Variablen gehen in die Versuchsbedingungen die Lehr- und Lernformen ein, als abhängige Variable gilt auf Seiten der Lerner in erster Linie der individuelle Lernerfolg. In geringerem Ausmaß finden auch Einstellungen, Denkstile, Motivationslagen und Beurteilungen der Lernsituationen durch die Lerner als abhängige Variablen Berücksichtigung. Versuchspersonen sind zumeist Schüler oder Studenten, deren Lernerfolg im Anschluss an die Lernphase als Klausurnote, Testscore oder Prüfungsleistung gemessen wird.

Bei Durchsicht der empirischen Ergebnisse zur Evaluationsforschung im mediendidaktischen Bereich (vgl. die Auflistung in Schulmeister 2002, S. 387f.) zeigen sich zwei generelle Tendenzen. Auf der einen Seite herrscht eine Unterteilung nach verschiedenen Lernsituationen in den Versuchs- und Kontrollgruppen vor. Die Versuchsleiter verteilen die Lerner per Zufall auf die Versuchsgruppen, um alle Lernvariablen im Einfluss konstant zu halten. Im Ergebnis fallen die Unterschiede zwischen den Lerngruppen trotz ausgefeilter statistischer Methoden nicht ins Gewicht. Die Vielfalt der Lernstile, Einstellungen und Erwartungen unter den Lernern nivellieren die Unterschiede in der Wir- 
kung der unabhängigen Variablen und den verschiedenen Lernsituationen. Auf der anderen Seite gelangt man zu pädagogisch interessanten und statistisch signifikanten Ergebnissen, sobald verschiedene Lernsituationen in Hinsicht auf didaktische Aspekte (z.B. Art der Instruktion im Programm, Ausmaß der Einwirkungsmöglichkeiten durch den Nutzer u.a.) deutlich voneinander abgegrenzt werden und außerdem die diesen unterschiedlichen Lernsituationen ausgesetzten Personen hinsichtlich spezifischer Lernvariablen in Gruppen (z.B. intrinsisch oder extrinsisch motiviert, ganzheitliches oder analytisches Denken u.a.) eingeteilt werden.

Diese beiden gegenläufigen Tendenzen in der Evaluationsforschung gelten für virtuelle wie für nicht-virtuelle Lernformen. Der amerikanische Psychologe Thomas L. Russell hat sich ausführlich mit dem „No Significant Difference Phenomenon“ (Russell 1999) in der empirischen Pädagogik und Psychologie befasst und zwei Websites zu diesem Thema eingerichtet. Die eine [http://tele education.nb.ca/significantdifference/]² beinhaltet Abstracts von anglo-ameri- kanischen Wirkungsstudien, die zu signifikanten Ergebnissen geführt haben, die andere Website führt eine Reihe von Studien auf, die zu nicht signifikanten Ergebnissen führten [http://teleeducation.nb.ca/nosignificantdifference/] ${ }^{3}$. Ende Oktober 2003 betrug das Verhältnis von nicht-signifikanten zu signifikanten Studien 124 zu 51. Die Studien reichen bis in die 1970er Jahre zurück. Die neueren Abstracts beziehen sich überwiegend auf virtuelle, telemediale Lernformen. Auf seiner alten Website hatte Russell Studien aufgelistet, die bis in die dreißiger Jahre des letzten Jahrhunderts reichten. Über 300 davon hatten zu nicht-signifikanten Ergebnissen geführt. Russell kommt zu dem Schluss, dass sich mit empirischer Forschung auf keinen Fall belegen lasse, dass sich eine bestimmte Unterrichtsmethode oder eine bestimmte Lernform grundsätzlich, gänzlich losgelöst von den spezifischen Voraussetzungen und Einstellungen der Lernenden, als anderen überlegen erweist.

Mit Bezug auf die mediendidaktische Evaluation von Lernen mit Software bedeutet das: Es ist verfehlt, allein Lernsituationen miteinander zu vergleichen, etwa Online-Lernen und Präsenzlernen. Derartige Studien führen zwangsläufig zu nicht-signifikanten Ergebnissen (vgl. z.B. Carey 2001). Aus dem Darbietungsmodus der Lerninhalte und der Unterscheidung zwischen verschiedenen Lernmedien sind keine Unterschiede im objektiv messbaren individuellen Lernerfolg oder der von den Lernern subjektiv empfundenen Lernqualität ableitbar. Die Evaluationsforschung muss sich vielmehr darauf konzentrieren, die Beziehung zwischen typischen Lernervariablen einerseits sowie didaktischen Aspekten von Lernsoftware andererseits zu untersuchen. Dazu ist es notwendig, ein auf die Eigenheiten der Lerner zentriertes Evaluationsdesign zu entwickeln, das unterschiedliche Lerntypen anhand diverser kognitiver, motivationaler und kul-

2 Abruf am 28.10.2003.

3 Abruf am 28.10.2003. 
tureller Variablen differenziert (vgl. z.B. das Modell in Richardson/Turner 2000).

Dieses Vorgehen birgt allerdings auch Gefahren in sich. Je spezifischer die Lernervariablen gefasst werden, desto geringer ist die Chance für Ergebnisse, die sich auf Lernsituationen jenseits der Evaluationsuntersuchung verallgemeinern lassen. Tatsächlich ist eine Tendenz zu einer „hochgradigen Differenzierung im Variablenbereich“ (Schulmeister 2002, S. 396) bei der Multimedia-Evaluation unverkennbar. Die Vielfalt der lernrelevanten Variablen (Vorwissen, Vertrautheit mit dem Programm, Denkstile, Kooperationsfähigkeit, Motivationslagen u.a.) auf Seiten der Lerner ist unendlich. Für die Seite der Programme, für ihre Usability und Didaktik, gilt das gleiche.

Betrachtet man die Fülle an Evaluationsstudien, die es, besonders im anglo- amerikanischen Raum, in der empirischen Medienforschung im Bereich virtuellen Lernens gegeben hat, fallen aber auch konstante Phänomene in den Ergebnissen und gleichförmige Wirkungen häufig untersuchter Variablen auf. Richardson und Turner (2000) berichten, dass sich in den gängigen demografischen Variablen wie Alter und Geschlecht immer wieder die gleichen Unterschiede bei der Beurteilung von Lernsoftware zeigen. Jüngere Personen und Männer beurteilen Software grundsätzlich positiver als ältere Personen bzw. Frauen. Außerdem bewerten analytische Denker, die Probleme abstrakt zergliedern, grundsätzlich virtuelle Lernumgebungen positiver als holistische Denker. Und für jüngere Studenten gilt in besonderem Maße, dass sie als Lerner hoch interaktive Lernprogramme gegenüber passiven, lesenden Lernformen am Computer deutlich bevorzugen.

Weiterhin spielen kognitive Variablen, die sich auf das Ausmaß an bewusster Selbststeuerung und Selbstkontrolle beim Lernen beziehen, eine entscheidende Rolle beim Lernerfolg wie bei der Beurteilung von Lernsoftware. Variablen wie „internal locus of control“ vs. „external locus of control“ oder „field dependency“ bzw. „field independenсу“ führen zu Unterschieden bei den entsprechenden Versuchsgruppen, wenn virtuelle Lernumgebungen beurteilt werden. Dabei gilt, je mehr sich die entsprechenden Personen ihrer eigenen, beim Lernen verwendeten kognitiven Stile auch bewusst sind, desto eher haben sie positive Lernerlebnisse, wenn sie sich zum Lernen mit dem Computer entscheiden (vgl. Summerville 1999).

\section{Der MLBK als Beispiel eines komplexen Lehr-Lern-Szenarios}

Der Methodenlehre-Baukasten stellt ein umfangreiches multimedial gestütztes LehrLern-Szenario dar, an dem verschiedene Personengruppen (Lerner, Autoren und Lehrende, die das fertige Softwareprodukt einsetzen) beteiligt sind. Alle liefern einen Beitrag zur Didaktik des Gesamtsystems und zur Ausprägung individuell messbarer Variablen des Lernens wie objektiver Lernerfolg oder subjektive Lernzufriedenheit. Evaluationsuntersuchungen können an einzelnen 


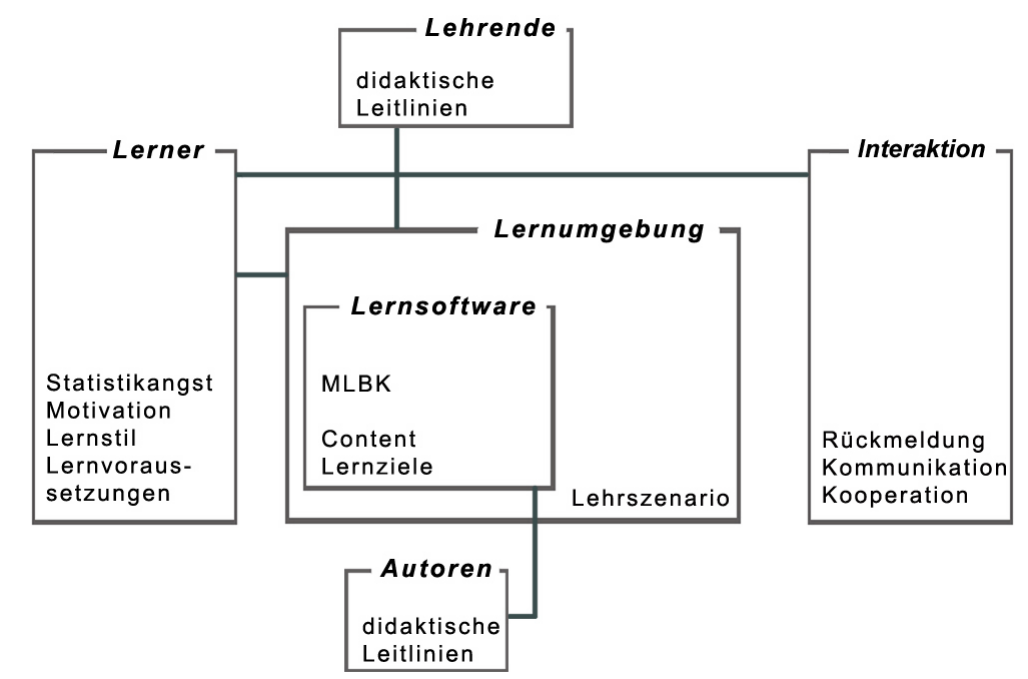

Abb. 2: Lehr-Lern-Modell im MLBK.

Aspekten dieses Szenarios, den Akteuren oder dem Softwareprodukt oder auch an der Interaktion mehrerer Aspekte ansetzen.

Aufgrund der oben referierten Untersuchungen zur Evaluation von Lernsoftware und der Untersuchungen zum Konstrukt Statistik-Angst ging unsere Vermutung vor Beginn der Evaluation dahin, dass es nicht möglich sein wird, allgemeine Aussagen über den Erfolg des Systems und den Lernerfolg zu treffen, sondern nur differenziert für unterschiedliche Studierendengruppen. Deshalb konzentriert sich die Evaluation im MLBK darauf, verschiedene Nutzergruppen und Lernszenarien miteinander zu vergleichen.

Wir erwarten, dass die Lernerfolge höher und die subjektiven Beurteilungen der mit dem MLBK anvisierten Zielgruppe deutlich positiver ausfallen und im statistischen Sinn auch signifikant werden, wenn man sie mit Lerngruppen vergleicht, die dazu eher den Gegenpol bilden. Der Evaluationsansatz des Methodenlehre-Baukastens ist also ebenso zielgruppenspezifisch wie die Didaktik. Zudem orientieren wir uns an der Ansicht von Zimmer und Psaralidis (2000), dass nur aus der Sicht der Lerner die Qualität einer Lernsoftware beurteilt werden kann. Zimmer und Psaralidis konstatieren, dass „die Qualität erst im Prozess des Lernens von den Lernenden selbst hergestellt wird“, deshalb könne es „keinen kausalen Zusammenhang zwischen objektiven Merkmalen und subjektiven Lernerfolgen geben“ (S. 265, im Original kursiv).

Für die Evaluation von Multimedia-Produkten ist zudem die Unterscheidung zwischen den Variablen Lernerfolg und Lernerzufriedenheit entscheidend. Beide Bereiche sind im Evaluationsdesign unabhängig voneinander zu erfassen. Der häufig in objektivierten Maßen, etwa Klausurnoten oder Prüfungsleistungen, erfasste Lernerfolg sagt nichts über die subjektive Zufriedenheit des Nut- 
zers mit der Lernsoftware aus (vgl. Kerres 2001 zu dieser Unterscheidung). Lernerfolg ist zudem ein hoch komplexes, widersprüchliches Merkmal. Niedrige Werte in „objektiven“ Lernerfolgsmaßen können mit positiven Beurteilungen der Lernsoftware durch einen Nutzer einhergehen und umgekehrt. Im Evaluationsansatz des MLBK gilt dem Faktor Lernerzufriedenheit das Hauptaugenmerk.

\subsection{Die Lernertypologie im Evaluationsansatz des MLBK}

Auf Seiten der Lerner wird eine Gruppierung hinsichtlich der Variablen Statistikangst, Motivation, Lernvoraussetzungen sowie Lernstil vorgenommen, wobei die letztere Variable durch einen Fragebogen zur Statistikangst, die ersten drei zusammengefasst durch einen anderen Fragebogen zum allgemeinen Lernverhalten erhoben werden. Das Motiv für die Entwicklung des Methodenlehre- Baukastens basiert auf der Erfahrung, dass die Studierenden vor allem in den geistes- und sozialwissenschaftlichen Fächern eine starke Abneigung zeigen, Statistik zu lernen (vgl. oben). Es wird versucht, das Konstrukt Statistik-Angst operationalisiert zu erfassen und dessen spezifische Wirkung als intervenierende Variable für den Lernprozess zu identifizieren. Die Erhebung erfolgt durch einen selbst konstruierten Fragebogen (Martens 2003) unter Berücksichtigung der Skalen der Arbeitsgruppe von Prenzel am Institut für Pädagogik der Naturwissenschaften Kiel (Prenzel/Kramer/Drechsel 2002). Prenzel et al. stützen sich auf die Selbstbestimmungstheorie von Deci und Ryan (1993). Diese unterscheiden intrinsisch versus extrinsisch motiviertes Verhalten, den Grad der Selbstbestimmung bei einer Tätigkeit und die Autonomie- versus Kontrollorientierung als individuelle Persönlichkeitseigenschaften.

Ein noch unveröffentlichter Pretest mit über 300 Studierenden erbrachte ein vorhersagekonformes Ergebnis. Anhand einer Latent-Class-Analyse ließen sich zwei Klassen, d.h. zwei Subpopulationen, aus den 300 Studierenden gewinnen. Der erste Typ (61\%) steht der Statistik eher positiv gegenüber (weniger Vorurteile), hat weniger Befürchtungen, erwartet positive Ergebnisse seiner Anstrengungen und traut sich selbst etwas zu (Kompetenzerwartung). Der zweite Typ (39\%) hat dagegen Befürchtungen und Vorurteile bezüglich der Statistik, erwartet weniger, traut sich selbst weniger zu und ist geneigt, eher aufzugeben. Es wird erwartet, dass unser didaktischer Ansatz im MLBK besonders diesen zweiten Typ anspricht.

Die weitere Unterscheidung erfolgt nach Lernstilen. Die Lernstile nach Kolb (Learning Style Inventory), welche erstmals 1976 (LSI1) und überarbeitet 1985 (LSI2) publiziert wurden, sind eine der bekanntesten Unterscheidungen in der Lehr-Lernforschung, deren Validität in etlichen empirischen Studien untersucht wurde (vgl. den Überblick bei Willcoxon/Prosser 1996). Lernen ist nach Auffassung von Kolb ein ständig fortschreitender Prozess, der sich durch Erfahrungen generiert (Kolb 1984). Er unterscheidet insgesamt vier Lernstile. Zwei 
geben an, wie Erfahrungen gesammelt werden, und die beiden anderen, wie die Erfahrungen anschließend verarbeitet werden (vgl. Abbildung 3). Auf der y-Achse finden sich die Pole der Erfahrungssammlung (von abstrakt bis konkret), auf der x-Achse die Pole der Erfahrungsverarbeitung (von experimentierend bis reflektierend). Kolb stellt dann vier Grundtypen auf, denen er eine Reihe von Eigenschaften sowie bestimmte Stärken und Schwächen zuschreibt (vgl. Kolb 1984; Smith/Kolb 1986). Die Lerntypen ergeben sich dann jeweils aus einer spezifischen Kombination, wie Erfahrungen gesammelt und anschließend verarbeitet werden.

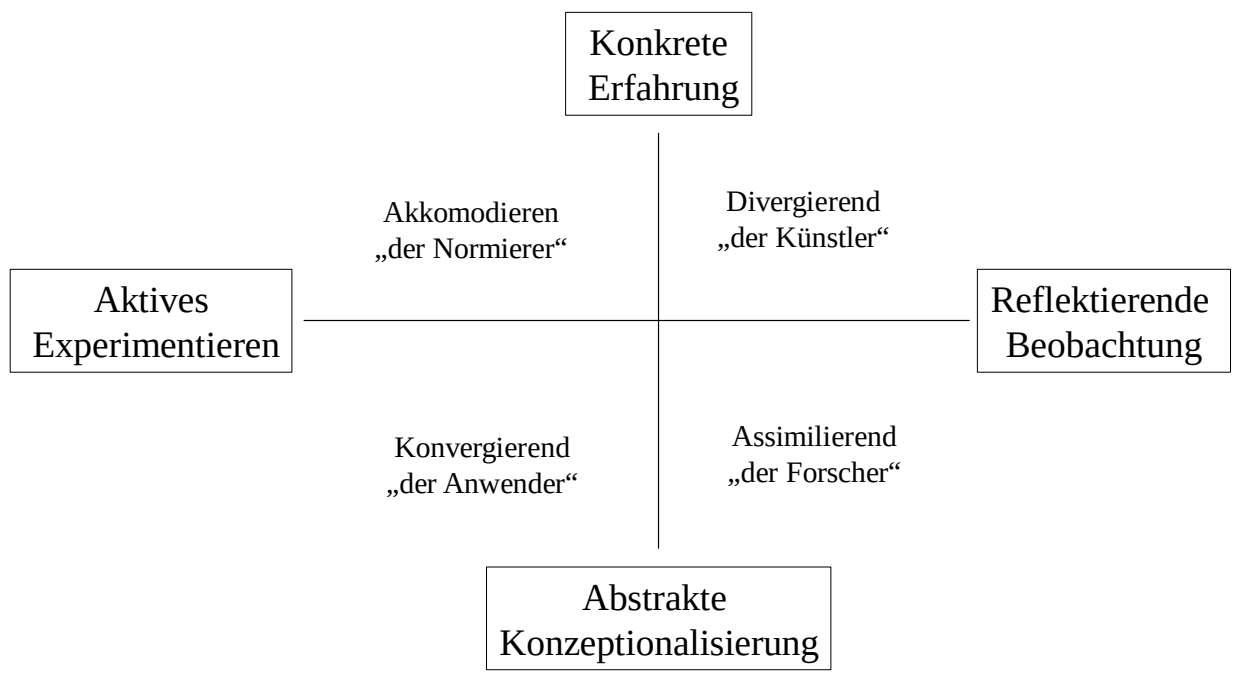

Abb. 3: Lernstile und Lerntypen nach Kolb.

Kolb beschreibt als erstes den divergierenden Typ (Divergierer), dessen bevorzugte Lernstile die konkrete Erfahrung und die reflektierende Beobachtung sind. Dieser Typ stellt die Zielgruppe des MLBK dar. Auf ihn ist die Didaktik zugeschnitten. Der zweite, der sog. konvergierende Typ (Konvergierer), verfügt über die Lernstile der abstrakten Konzeptionalisierung und des aktiven Experi- mentierens. Diese Personen bevorzugen Lernaufgaben, in denen es nur eine korrekte Lösung gibt. Für diese Personengruppe, so unsere Annahme, reicht die herkömmliche Statistikausbildung. Der Typ des assimilierenden Lerners (Assi- milierer) lernt durch analytisches Begreifen und reflektierendes Beobachten. Auch dieser Typ zeichnet sich durch Stärken in der deduktiv geführten Statistiklehre aus. Auch ihn rechnen wir unserer Annahme gemäß nicht zur Zielgruppe des MLBK. Der vierte Typ, der Akkomodierer (akkomodierend, konkretes Erfahren und aktives Experimentieren), ist wiederum wie der erste Typus gut geeignet für die Didaktik des MLBK. Für den ersten und den vierten Typ erwarten wir hohe Werte für die Variablen Lernerfolg und Zufriedenheit mit dem MLBK, für die Typen zwei und drei dagegen eher niedrige Werte.

Die Messung der Lernstile erfolgt durch eine modifizierte Version des Lernstilinventars von Kolb (Wolff 2003). In unserer Modifizierung werden kultur- 
bedingte Anpassungen im Hinblick auf den Einsatz des amerikanischen Fragebogens im europäischen Raum eingearbeitet. Er setzt sich aus den vier Skalen Konkretes Erfahren, Reflektierendes Beobachten, Abstraktes Konzeptualisieren und Aktives Experimentieren zusammen (s. Abbildung 3). Auch mit dem Lernstilfragebogen fand ein Pretest mit über 400 Studierenden statt. 364 der 400 Probanden aus der Voruntersuchung lassen sich eindeutig einem der Lerntypen zuordnen: 87 der Gruppe der Divergierer, 93 der Gruppe der Assimilierer, 59 der Gruppe der Konvergierer und 125 der Gruppe der Akkomodierer. Unsere erwartete Zielgruppe (Divergierer und Akkomodierer) addiert sich damit auf 212 Studierende von 400 Befragten. Von den ursprünglich 48 in die Voruntersuchung eingegangenen Items (zwölf pro Skala) wurden aufgrund der Itemanalyse 16 Items herausgenommen, so dass der Fragebogen nun insgesamt 32 Items (acht Items pro Skala) enthält.

\subsection{Zur grundsätzlichen Bedeutung von Lernertypologien}

Typologien von Lernern in der Pädagogik sind, ähnlich wie Persönlichkeitstypologien in der Psychologie, ideelle Abstraktionen. Die Unterscheidungen der Typen gelten nicht für die exakte Abgrenzung einzelner Individuen voneinander. Jeder Mensch trägt Mischformen in sich. Typologien stellen Zusammenfassungen vielfältiger Wirkungen zwischen Faktoren innerhalb eines komplexen Systems dar. Im Fall der Lernstile nach Kolb ist dies das überindividuell mögliche Lernverhalten in verschiedenen Situationen mit unterschiedlichen Lernstoffen. Typologien haben in der empirischen sozialwissenschaftlichen Forschung heuristische Funktionen. Sie dienen den Forschenden als Orientierung innerhalb des von ihnen untersuchten Wirklichkeitsbereiches und fassen diskursive Wissensbestände im jeweiligen Forschungsgebiet zusammen. Nur auf der Basis qualitativer Unterscheidungen wie Typologien sind in der empirischen pädagogischen wie psychologischen Forschung überhaupt quantitative Messungen von Variablen und die Konstruktion quantitativer Zusammenhänge und Abhängigkeiten zwischen Variablen sinnvoll.

Auch wenn die Lernstile von Kolb auf Personen bezogen beschrieben wurden, bedeutet das nicht, dass der individuelle Lernprozess allein auf den persönlichen, inneren Ressourcen eines Menschen beruht. Lernen ist immer das Ergebnis einer Anpassung von Individuum und Umwelt. Gute Lerner mit Lernkompetenzen in unterschiedlichen Fächern sind zumeist flexible Lerner, die potenziell über alle vier von Kolb unterschiedenen Lernstile verfügen und sich damit erfolgreich an verschiedene Lerngegenstände anpassen können.

Was grundsätzlich für Typologien in den Humanwissenschaften gilt, trifft auch auf Typologien von Lernstilen zu, nämlich dass Typologien mit einem hohen Abstraktionsgrad viele verschiedene Lebenssituationen integrieren. Sie geraten daher sehr allgemein. Unterschiede zwischen den Typen in der Wirkung von gemessenen Variablen sind dann oft schwer nachzuweisen. Typologien mit 
einem niedrigen Abstraktionsgrad sind dagegen spezifischer. Sie differenzieren leichter zwischen Personen, was sich auch in der Messung von Variablenunterschieden niederschlägt. Typologien mit einem niedrigen Abstraktionsgrad haben aber den Nachteil, dass Untersuchungsergebnisse, die auf Basis dieser Typologie gewonnen wurden, schwer zu verallgemeinern sind. Befunde, die an Stichproben gewonnen wurden, die auf Typologien mit hohem Abstraktionsgrad beruhen, sind dagegen eher auf andere Lebenssituationen übertragbar.

Die Typologie der Lerner von Kolb weist einen hohen Abstraktionsgrad auf. Sie integriert empirisch gut bewährte Typologien aus der Kreativitätsforschung (divergierendes vs. konvergierendes Denken), der Wahrnehmungspsychologie (analytisch vs. holistisch, bei Kolb aufgehoben in der Unterscheidung von abstrakter Konzeptualisierung gegenüber konkreter Erfahrung) und der Lernpsychologie (aktives vs. passives Lernverhalten). So sind Vergleiche der Ergebnisse der Evaluation im MLBK mit anderen Evaluationsstudien, soweit diese typolo- gische Unterscheidungen der Lerner bzw. Nutzer beinhalten, möglich.

\subsection{Der Evaluationsansatz des MLBK im Überblick}

Das Hauptgewicht im komplexen Lehr-Lern-Szenario des MLBK (s.o. Abbildung 1) liegt auf der Untersuchung der Lerner, den wichtigsten Akteuren in diesem Szenario. Wie zufrieden die Lerner mit dem MLBK sind, wird anhand einer Usability-Befragung mit Hilfe des EMIL-Fragebogens (Zehner 2003) erhoben. Der davon unabhängige Lernerfolg wird mittels eines selbst entwickelten Fragebogens (Mühlenfeld 2003) gemessen. Alle Befragungen finden online statt. Zu Anfang müssen die Studierenden sich einen Code zusammenstellen, der sicherstellt, dass der Student auch bei der zweiten Befragung mit den Ausgangsdaten in Verbindung gebracht werden kann. Eine vorab vergebene Identifikationsnummer gewährleistet eine Zuordnung zum jeweiligen Lernszenario. Eine Identifizierung einzelner Studierenden ist an Hand des Codes nicht möglich. Im Übrigen finden für alle Daten die Auflagen des Bundesdatenschutzgesetzes (BDSG) Anwendung.

Weitere Aspekte des Lehr-Lern-Szenarios sind im Evaluationsdesign mit einbezogen. Mit in die Evaluation wird auch die Entwickler- und Autorenperspektive aufgenommen. Wie die Autoren ihre Arbeit bewältigen, welche Strategien sie dazu entwickeln und auf welches Wissen sie dabei zurückgreifen, also das Zusammenführen von theoretischen und praktischen Wissensbeständen, wird im amerikanischen Sprachraum als „personal practical theories“ (Pan/Deets/Phillips/Cornell 2003) bezeichnet. Die Untersuchung dieser „personal practical theories“ bei den Autoren des MLBK findet anhand von case studies statt (Gücker 2003).

Bei den Lehrenden erfolgt eine Unterscheidung nach verschiedenen Lehrszenarien, die als unabhängige Variable kontrolliert werden sollen, um eindeutige Aussagen über Zusammenhänge zwischen Lernervariablen und Lehrszenarios 


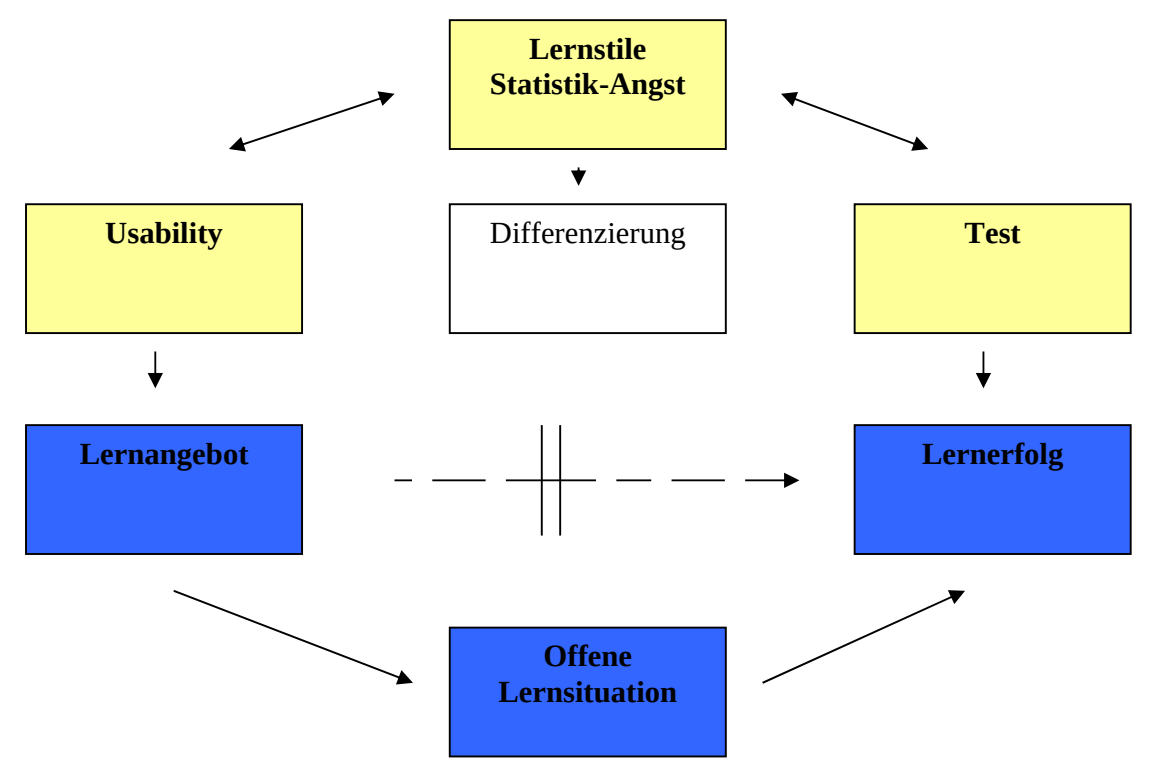

Abb. 4: Schwerpunkte der Evaluation im MLBK.

sowie unserer Lernsoftware, dem MLBK, treffen zu können. Die einzelnen Lernszenarien werden danach festgehalten, wie die Lehrenden beabsichtigen, den MLBK im Unterricht einzusetzen. Es werden eine vorlesungsbegleitende Variante (die Studierenden können den MLBK zusätzlich zur Vorlesung im Selbststudium nutzen), eine tutorielle Variante (die Studierenden nutzen den MLBK im Computerraum in Kleingruppen mit Tutoren) sowie eine Dozen- ten-zentrierte Variante (der Dozent setzt den MLBK in der Vorlesung ein) unterschieden.

In Bezug auf die Ergebnisse der Evaluation ist unsere Hypothese, dass die Unterscheidung der Lerner (nach der Typologie von Kolb und der Unterscheidung hinsichtlich Statistik-Angst) die Ergebnisse der Bewertung verschiedener Lehrszenarien durch die Nutzer determiniert. Die beiden Lerntypologien definieren den gesamten Untersuchungsrahmen.

In Abbildung 4 ist der Ansatz unserer Evaluation zusammengefasst. Ausgangspunkt unserer Überlegungen ist die Tatsache, dass viele Lerner mit den herkömmlichen Lernangeboten zur Statistik nicht zurechtkommen (in Abbildung 4 durch Doppelstrich symbolisiert). Wir versuchen daher, durch Bereitstellung einer offenen Lernsituation durch geleitetes entdeckendes Lernen eine Didaktik zu verwirklichen, die es dem einzelnen Lerner gemäß seiner kognitiven Struktur erlaubt, seinen eigenen Weg zum Verständnis der Statistik zu gehen. Im Evaluationsdesign wird bei den Lernenden eine Differenzierung vorgenommen, indem nach Ausprägungen der Statistik-Angst und den kognitiven Lernstilen unterschieden wird. Die Zufriedenheit mit dem Lernangebot wird mittels des Usability-Fragebogens erhoben, der Lernerfolg mit Hilfe eines Online-Tests abgefragt. Schließlich werden Zusammenhänge zwischen Zufriedenheit und Lernerfolg mit Statistik-Angst und kognitiven Lernstilen hergestellt (vgl. Abbildung 4). 
Die Evaluation im MLBK orientiert sich an generellen Konzepten zur Untersuchung telemedialen Lernens. Fricke (1995) postuliert unter Rückgriff auf die Evaluationsmodelle von Ross und Morrison (1995) sowie Seidel und Park (1994) eine gleichmäßige Berücksichtigung von Instructional Conditions (Lernervariablen und Lernstoffeinflüsse), Instructional Methods (der Wirkung der Lernumgebung in ihren virtuellen und nicht-virtuellen Anteilen) sowie der Instructional Outcomes (der mit dem Einsatz der Software intendierten Lernziele). Zwischen Instructional Methods und Instructional Outcomes bestehe eine Wechselwirkung. Deskriptiv lassen sich Lernziele aus den Besonderheiten der Lernumgebungen ableiten. Häufig sind aber schon in der Planungsphase der Software mit der Bestimmung von Zielgruppen präskriptiv pädagogische Ziele für das Design handlungsleitend.

Der MLBK steht unter der URL http://www.methodenlehre-baukasten.de zur freien Verfügung für Lernende und Lehrende. Es finden ständig noch Entwicklungsarbeiten statt, um fehlende Übungen zu ergänzen oder fehlerhafte zu verbessern oder Teile in Modulen, mit denen wir noch nicht zufrieden sind, zu ersetzen. Rückmeldungen der Nutzer sind uns sehr erwünscht, um diesen Entwicklungsprozess voranzutreiben. Für die Zukunft wird ein Geschäftsmodell erarbeitet, das die Wartung, Pflege und Weiterentwicklung des Systems tragen soll und für die nachhaltige Bereitstellung des Systems sorgen kann.

\section{Literatur}

Abel, J.: Probleme der Ausbildung in Empirie und Statistik an erziehungswissenschaftlichen Fachbereichen. Empirische Pädagogik, 4 (13), 1999, S. 355-369.

Bruner, J.: The act of discovery. Harvard Educational Review, 31, 1961, S. 21-32.

Carey, J. M.: Effective Student Outcomes: A Comparison of Online and Face-to-Face Delivery Modes. 2001 [http://www.ed.psu.edu/acsde/deos/deosnews/deosnewsll_9.asp] (Abruf am 04.09.2003).

Deci, E. L.lRyan, R. M.: Die Selbstbestimmungstheorie der Motivation und die Bedeutung für die Pädagogik. Zeitschrift für Pädagogik, 2, 1993, S. 223-238.

Dreger, R. MJ Aiken L. R.: The identification of number anxiety in a college population. Journal of Educational Psychology, 48, 1957, S. 344-351.

Fricke, R.: Evaluation von Multimedia. In: Issing, L.J./Klimsa, P. (Hrsg.): Information und Lernen mit Multimedia. Weinheim 1995, S. 400-413.

Gruber, H./Renkl, A.: Alpträume sozialwissenschaftlicher Studierender: Empirische Forschungsmethoden und Statistik. In: Lompscher, J./Mandl, H. (Hrsg.): Lehr-Lernprobleme im Studium - Bedingungen und Veränderungsmöglichkeiten. Bern 1996.

Gücker, R.: Complex statistical problems and guided Discovery Learning: The tasks of the instructional designer. Proceedings of the 4th international conference on information technologies in education. Samos 2003.

Gücker, R./Nuyken, K./Vollmers, B.: Entdeckendes Lernen als didaktisches Konzept in einem interdisziplinären Lehr-Lernprogramm zur Statistik. In: Kerres, M./Voß, B. (Hrsg.): Digitaler Campus. Vom Medienprojekt zum nachhaltigen Medieneinsatz in der Hochschule. Münster 2003, S. 250-259.

Heemskerk, J.: „Statistikphobie“ - Struktur negativer Einstellungen zur Methodenausbildung bei Studenten der Sozial- und Erziehungswissenschaften. Psychologie in Erziehung und Unterricht, 22, 1975, S. 65-77.

Heller, R. S.: The Role of Hypermedia in Education: A Look at the research issues. Journal of Research on Computing in Education, 4 (22), 1990, S. 431-441. 
Hunsley, J.: Cognitive Processes in Mathematics Anxiety and Test Anxiety: The Role of Appraisals, Internal Dialogue, and Attributions. Journal of Educational Psychology, 79, 1987, S. 388-392.

Jugendwerk der Deutschen Shell (Hrsg.): Jugend 97. Zukunftsperspektiven, gesellschaftliches Engagement, politische Orientierungen. Opladen 1997.

Kerres, M.: Multimediale und telemediale Lernumgebungen. 2. Auflage, München 2001.

Kolb, D. A.: Experiental Learning. Englewood Cliffs 1984.

Kolb, D. A.: Learning Style Inventory. Boston 1985.

Martens, L: Statistik-Angst Test. Institut für Psychologie der Universität Bremen 2003.

Moallem, M,: Applying Constructivist and Objectivist Learning Theories in the Design of a Web-Based Course: Implications for Practice. Educational Technology and Society, 4 (3), 2001.

Mühlenfeld, U.: Lernerfolgstest. Institut für Soziologie der Universität Bremen 2003.

Pan, S./Deets, J./Phillips, W./Cornell, R: Pulling tiger's teeth without getting bitten. Instructional Designers and Faculty. The Quarterly Review of Distance Education, Volume 4(3), 2003, S. 289-302.

Prenzel, M./Kramer, K/Drechsel, B.: Self-determined and interested learning in vocational education. In: Beck, $K(\mathrm{Ed}$.$) : Teaching - learning processes in vocational education. Foundations of modern$ training programs. Frankfurt 2002, S. 43-68.

Neber, H. (Hrsg.): Entdeckendes Lernen. 2. Auflage, Weinheim 1975.

Piaget, J.: Genetic Epistemology. New York 1970.

Renkl, A.: Wer hat Angst vorm Methodenkurs? Eine empirische Studie zum Streßerleben von Pädagogikstudenten in der Methodenausbildung. In: Olechowski, R/Rollett, B. (Hrsg.): Theorie und Praxis. Aspekte empirisch-pädagogischer Forschung - quantitative und qualitative Methoden. Frankfurt 1994, S. 178-183.

Richardson, J. A./Turner, A.: A Large-Scale local evaluation of students learning experiences using virtual learning environments. Educational technology and Society, 3 (4), 2000.

Ross, St. M./Morrison, G. R.: Evaluating interactive media: Issues and suggested practices. In: Schenkel, P. (Hrsg.): Qualität und Evaluation von Lernsoftware. Nürnberg 1995.

Russell, T.L.: The No Significant Difference Phenomenon. Chapel Hill, NC: Office of Instructional Telecommunications, North Carolina State University 1999.

Schulmeister, R: Angst vor Statistik. Empirische Untersuchungen zum Problem des Statistik-Leh- rens und Lernens. Hamburg: Arbeitsgemeinschaft für Hochschuldidaktik 1983.

Schulmeister, R: Grundlagen hypermedialer Lernsysteme. 3., korr. Auflage. München 2002. Schulmeister, R.: Zur Komplexität problemorientierten Lernens. In: Asdonk, J./Kroeger, H./Strobl,

G./Tillmann, K-J./Wildt, J. (Hrsg.): Bildung im Medium der Wissenschaft. Zugänge aus Wissenschaftspropädeutik, Schulreform und Hochschuldidaktik. Weinheim 2002a.

Schulmeister, R.: Didaktisches Design aus hochschuldidaktischer Sicht. Ein Plädoyer für offene Lernformen. In: Meister, D./Rinn, U. (Hrsg.): Didaktik und neue Medien. Münster 2003, S. 19-49.

Seidel, R. J./Park, O.: An historical perspective and a model for evaluation of intelligent tutoring systems. Journal of Educational Computing Research, 10, 103-128, 1994.

Smith, D. M./Kolb, D. A.: User's guide for the Learning Style Inventory. A manual for teachers and Trainers. Boston 1986.

Stark, R./Mandl, H: Probleme der Methodenausbildung. Analyse und Intervention aus motivationspsychologischer Perspektive. Forschungsberichte der Ludwig Maximilians Universität München, Nr. 116, 2000.

Summerville, J.: Role of awareness of cognitive style in hypermedia. International Journal of Educational Technology, 1 (Inaugural Issue), 1999.

Willcoxson, L./Prosser, M.: Kolb’s learning style inventory (1985): Review and further study of validity and reliability. British Journal of Educational Psychology, 66, 1996, S. 251-261.

Wolff J.: Lernstiltest. Institut für Psychologie der Universität Greifswald 2003.

Zehner, M. L.: Usability-Fragebogen (EMIL). Institut für Geographiestatistik der Universität Rostock 2003.

Zeidner, M.: Statistics and Mathematics Anxiety in Social Science Students: Some Interesting Parallels. The British Journal of Educational Psychology, 61, 1991, S. 319-328.

Zimmer, G./Psaralidis, E.: „Der Lernerfolg bestimmt die Qualität der Lernsoftware!“ Evaluation von Lernerfolg als logische Rekonstruktion von Handlungen. In: Schenkel, P./Tergan, S. O./Lott- mann, A. (Hrsg.): Qualitätsbeurteilung multimedialer Lern- und Informationssysteme. Evaluationsmethoden auf dem Prüfstand. Nürnberg 2000, S. 262-303. 
Das diesem Bericht zugrunde liegende Vorhaben wurde mit Mitteln des Bundesministeriums für Bildung und Forschung unter dem Förderkennzeichen 01NM108A gefördert. Die Verantwortung für den Inhalt dieser Veröffentlichung liegt bei den Autoren. 